Témoigner Témoigner. Entre histoire et mémoire

Getuigen Revue pluridisciplinaire de la Fondation Auschwitz

$121 \mid 2015$

Violences radicales en scène

\title{
Turquie - Istanbul, poste avancé mémoriel ?
}

Turkey: Istanbul as a memory outpost

\section{Philippe Mesnard}

\section{(2) OpenEdition}

\section{Journals}

Édition électronique

URL : https://journals.openedition.org/temoigner/3606

DOI : 10.4000/temoigner.3606

ISSN : 2506-6390

Traduction(s) :

Turkey - Istanbul as a memory outpost - URL : https://journals.openedition.org/temoigner/3611 [en]

Éditeur :

Éditions du Centre d'études et de documentation Mémoire d'Auschwitz, Éditions Kimé

Édition imprimée

Date de publication : 1 octobre 2015

Pagination : 186-191

ISSN : 2031-4183

Référence électronique

Philippe Mesnard, «Turquie - Istanbul, poste avancé mémoriel ? », Témoigner. Entre histoire et mémoire [En ligne], 121 | 2015, mis en ligne le 01 octobre 2016, consulté le 04 février 2022. URL : http:// journals.openedition.org/temoigner/3606; DOI : https://doi.org/10.4000/temoigner.3606 


\section{Turquile}

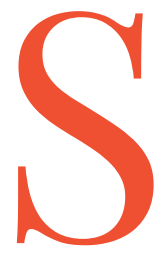

'il est vrai qu'en Europe ce que l'on entend par (histoire et) mémoire des violences extrêmes paraît être coupé du social, planant au-dessus de la société en la perdant régulièrement de vue, ce n'est pas le cas d'autres zones géographiques. Là, là-bas, les usages de la mémoire mettent en évidence un dynamisme de sociétés en mutation dont la poussée ne vient ni des politiques, ni des intellectuels, ni ne tient à eux. J'avais fait récemment ce constat en Argentine. J'y avais rencontré des membres d'associations de quartiers animés à la fois d'une vitalité et d'une exigence surprenantes où, aux remémorations des violences de la dictature, généralement sur les lieux mêmes où elles ont été exercées, se conjuguent aujourd'hui travail social et militantisme politique. Mutatis mutandis, c'est à un constat similaire que m'a conduit un récent voyage à Istanbul (fin juin 2015).

Ce sera donc là le sujet de ce papier qui va tenter de confirmer combien la société turque constitue aujourd'hui un véritable laboratoire mémoriel, dans un double sens. Parce que les questions de mémoire constituent un facteur déterminant dans la reconfiguration sociopolitique qui est en train de se réaliser en Turquie (sans présumer des teintes que cela recouvrera une fois cette reconfiguration stabilisée). Mais aussi en considérant qu'il y a là comme un miroir permettant de mieux objectiver les changements en cours del'Europe, son identité à construire avec ses identités nationales (puzzle qui peut virer à une coloration alarmante entre les séparatismes, les extrémismes et les radicalismes, et les réactions à ceux-ci).

Autre préalable, j’avais mis en avant dans la présentation du dossier du précédent numéro de la revue le risque d'entretenir une stigmatisation dont la « Turquie » et les « Turcs » ont souvent été l'objet; il faut voir là une des manifestations de ce qu'Edward Saïd a qualifié d' « orientalisme », exacerbée par la connotation négative que supporte aujourd'hui la société tout entière du fait qu'une partie d'elle-même et ses gouvernements successifs refusent de reconnaître qu'il y a effectivement eu, en 1915, une planification systématique de l'anéantissement des Arméniens vivant sur le territoire national à laquelle correspond précisément la qualification juridique de "génocide ». Car cette stigmatisation, par les effets inductifs que Goffman a clairement analysés, peut tout à fait stimuler le sentiment de cohésion nationale et, ce faisant, renforcer la doxa négationniste qui est, en l'occurrence, identitaire. Considérons plutôt qu'Istanbul, ville décentrée entre les rives occidentales et orientales du pays, puisse être, comme le suggère Mehmet Fatih Uslu de l'Université Şehir, une tête de pont du travail de mémoire 


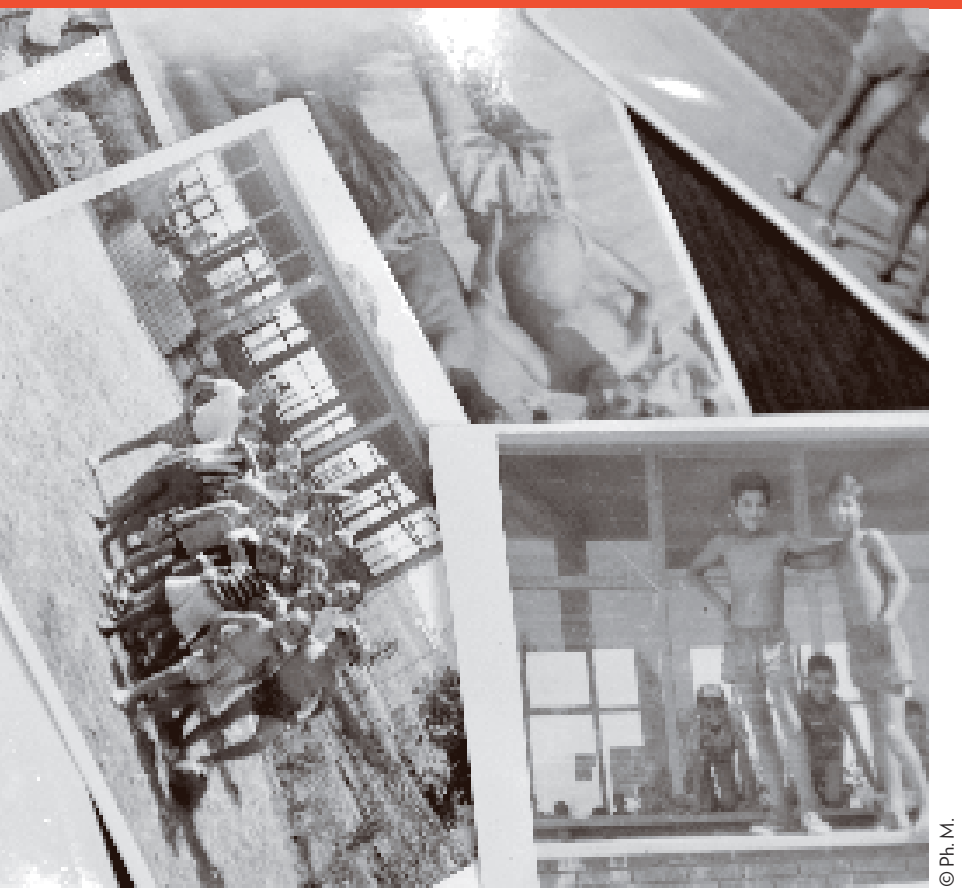

en Turquie concernant la reconnaissance du génocide arménien et touchant, par ricochet, une histoire où les minorités ont toutes été, à un moment ou à un autre, victimes de ségrégation, de répression, d'expulsion généralement accompagnées de massacres.

$$
\text { 次 }
$$

Malgré la faiblesse de l'actuelle communauté ne comptant, principalement installés à Istanbul, qu’environ 60000 membres - ce qui la range au nombre des hyperminorités -, la mémoire du génocide est présente à plus d'un niveau et ceux qui la revendiquent et militent pour sa reconnaissance sont extrêmement impliqués dans la vie même de cette société. Si cet aspect m'a frappé, c'est aussi parce que cette mémoire repose sur une interaction soutenue entre acteurs turcs et arméniens conduisant Michel Marian à parler de « mémoire partagée ».

Anadolu Kultur, la fondation Hrant Dink, DurDe (SOS Racisme), Helsinski Citizens Assembly, IHD (Associations des droits de l'homme) figurent parmi les associations, fondations et ONG engagées dans ce dur travail à contre-courant d'une culture dominante où la victime vulnérable, ni héros, ni martyr, n’a guère
_ Scène de la vie quotidienne

à Kamp Armen (1960).

de place. Je dirais même que cette imperméabilité est relativement autonome des raisons politiques qui sous-tendent la non-reconnaissance du génocide. Précisément, un des aspects qui font que la Turquie peut être considérée comme un véritable chantier mémoriel tient à ce qu’à travers des groupes comme ceux précédemment mentionnés ou des initiatives individuelles, se manifestent des volontés de se réapproprier une partie du passé dont la société a été privée pour des raisons éminemment politiques, certes, mais où la culture a joué un rôle déterminant.

En effet, la fondation de l'État moderne et de la République en 1922 - instaurant une rupture radicale avec le passé ottoman - a été portée par le Mouvement national turc à la tête duquel figuraient de nombreux membres du CUP, parti responsable du génocide. Non seulement cela a favorisé leur intégration dans l'appareil d'État, mais en tant que pères fondateurs, ils ont été célébrés comme des héros (Taner Akçam, 28). En 1926, le Parlement vote même une loi accordant terres et pensions aux familles d'anciens membres du CUP et, fait remarquable, y compris pour deux hauts responsables exécutés lors des procès du génocide qui ont eu lieu en 1920 et pour d'autres criminels qui avaient été exécutés par des Arméniens par vengeance. Non seulement cette glorieuse réhabilitation réécrivait l'histoire, mais, annulant la valeur exemplaire des procès, elle forgeait l'impunité en modèle, du moins pour les puissants et ceux qui avaient servi la cause de l'État, leur octroyant par là même une transcendance qui les plaçait au-dessus des lois, symboliquement, réellement et dans l'imaginaire national. Se réalisait là comme la ratification absolue du rapport entre fondation, violence radicale et oubli, cet oubli dont Renan disait sans détour en 1882 qu'il était, y compris quand il s'agit d'erreur historique (sic), « un facteur essentiel de la création d'une nation» (Renan, 13-14). Il est un autre facteur qui confère une extrême singularité à la relation que la Turquie moderne entretient avec son passé, rendant encore plus ardu le parcours qui ramènerait $\bullet \bullet$ 


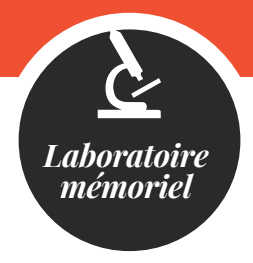

•. à celui-ci. Il s'agit d'une magistrale réforme alphabétique mise en place en 1928 à l'initiative de l'Atatürk. En imposant le passage d'une écriture ancestrale en caractères arabes aux caractères latins enrichis de signes diacritiques - scénario digne d'un roman d'Orwell -, le pouvoir politique rendait difficilement accessibles aux générations ultérieures les textes officiels, publics ou privés de l'histoire ottomane. Nul mieux qu'Emine Sevgi Özdamar, dans sa nouvelle « Mutterzunge » du recueil éponyme, a traduit l'expérience de cet écart :

J'apprendrail'Arabe, c'était autrefois notre écriture, après
notre guerre de libération en 1927, Atatürk l'interdit etles
lettres latines sont arrivées, mon grand-père connaissait
seulement l'écriture arabe, moije connaissais seulement
l'alphabet latin, c'est-à-dire que si mon grand-père et moi
étions muets et que nous ne pouvions nous raconter des
choses que par l'écriture, nous ne pourrions nous racon-
ter des histoires (p. 14).

Or, aujourd'hui, c'est-à-dire depuis un peu plus d'une dizaine d'années, le rapprochement entre les groupes qui s'opère dans la société permet l'émergence d'une histoire qui jusque-là ne pouvait avoir droit ni de cité, ni d'être lue facilement. Les histoires orales dévoilent (Uğur Ümit Üngör, 57-65, Marchand \& Perrier, 52-66) comme une généalogie autre à travers différents médiateurs dont la plupart se sont déclarés à partir du moment où Hrant Dink, fondateur de l'hebdomadaire Agos sur lequel nous reviendrons, a acquis une certaine visibilité dans le champ médiatique et après la prise de conscience que son assassinat, le 19 janvier 2007, polarisa.

$$
\text { 棌棌 }
$$

Nor Zartonk signifie « nouvelle renaissance », au sens d'un nouveau commencement et d'un réveil qui viendraient non pas prêcher un identitarisme arménien, mais faire entendre les voix arméniennes longtemps tues en Turquie tout en soutenant d'autres causes minoritaires. L'association a été fondée le 18 mars 2007, peu après la mort de Hrant Dink, explique Sayat, un de ses responsables. Très vite, arrive le mot

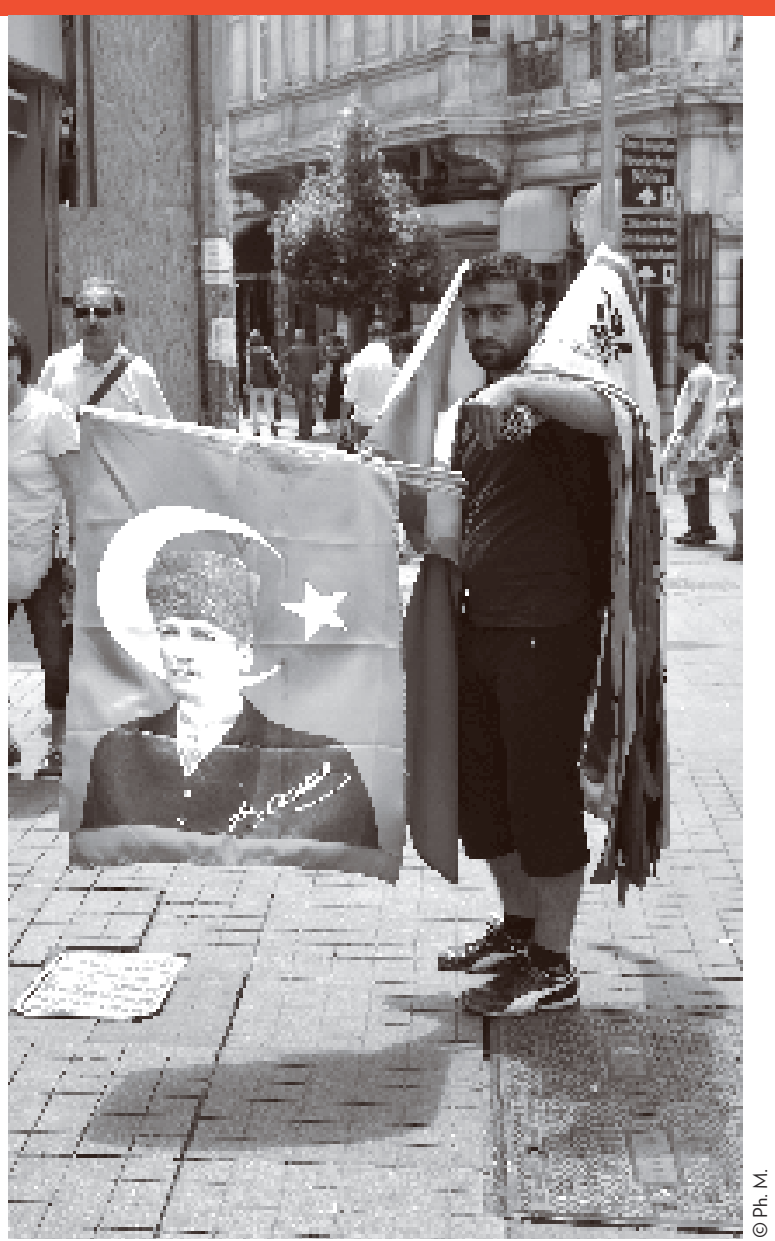

_ Dans une rue d'Istanbul (2015).

« activisme ». En effet, ce sont des militants qui, pas toujours bien vus par une communauté arménienne discrète et normative, affectionnent une phraséologie mi-libertaire, mi-marxiste et une étiquette anticapitaliste sur leur site (http://www.norzartonk.org/en). Tout en occupant une position centrale dans leurs activités, la revendication de la reconnaissance du génocide et le renouveau de la culture arménienne - dans les murs de l'association, on peut suivre des cours d'arménien - sont inscrits dans un ensemble de luttes contre l'exclusion, le racisme, la destruction écologique, l'homophobie et les violences contre les femmes. Ce sont là nos principes de base, dit Sayat, reconnaissant par ailleurs qu'il s'en faudrait de peu pour que les membres de Nor Zartonk ne soient menacés et risquent leur vie. Ce constat a la couleur d'un 
triste mélange où le romantisme se mêle au réalisme pragmatique d'une situation encore très exposée aux groupes ultranationalistes couverts par l'État. En 2015 encore, plusieurs attentats ont notamment ciblé des permanences de l'HDP, le Parti démocratique des peuples proKurdes, à Adana et Mersin, d'autres ont notamment été perpétrés à Diyarbakir.

À ce titre, sur le plan politique, les récents succès de l'HDP, dont Nor Zartonk se déclare proche, aux élections législatives de juin 2015 font percevoir des signes de changement. Ce parti soutient en effet la normalisation des relations entre Turquie et Arménie et la reconnaissance du génocide. Il est, de toute façon, difficile de prendre la mesure de la singularité des usages mémoriels en Turquie, sans considérer la place que tiennent les Kurdes, en particulier, et les minorités (juive, grecque, syriaque, chaldéenne...), en général. Les Kurdes ont une position singulière de tiers dans le processus de la reconnaissance alors qu'ils étaient fortement impliqués dans les crimes de 1915, comme s'ils avaient d'abord été les intermédiaires des assassins, leurs tiers autrement dit. En effet, regroupés en bandes ou escadrons, on sait qu'ils ont servi de bras criminels du génocide (le constat de ressemblance avec le rôle des bataillons ukrainiens en appui aux SS durant la Shoah est tentant). On leur reconnaît meurtres, viols et autres cruautés sans merci et pillages, rapts d'enfants et de femmes, mariages forcés. Cela jette aujourd'hui un grand trouble dans le rapport à la filiation d'une société dont la culture moderne ne souffrait pas les étrangers, et où les Kurdes avaient une position de subalterne, longtemps persécutés par un État centralisateur et jacobin.

Sous cet angle les questions de mémoire apparaissent différemment. Sans que leur importance en soit diminuée, elles se rangent sous la bannière de la résistance à l'ultraconservatisme d'une société qui commence lentement à s'ouvrir. Ainsi, la priorité passe d'abord par le vivre ensemble, et c'est à l'intérieur de ce qui se présente comme un projet de société que prend place et sens le repérage des sites détruits ou abandonnés pour les faire revivre, ou pour les préserver. Mais ces activités où se combinent revendication de reconnaissance et activisme social resteront longtemps encore comme la fragile tête de pont de ces change- ments dont le lieu d'émergence demeure prioritairement Istanbul.

Le litige dont le lieu d'hébergement de Kamp Armen reste l'enjeu est un exemple de la précarité de cette situation. Ce centre d'hébergement pour jeunes Arméniens venus d'Anatolie, par lequel est passé Hrant Dink, avait à l'origine été ouvert par Hrant Güzelyan, en 1958. Au début, il s'agissait de prendre en charge des orphelins - le « reste de l'épée », Kılıç artığı dit-on en turc pour désigner ceux qui avaient échappé aux massacres -, mais, par la suite, des parents y placèrent en pension leurs enfants pour qu'ils puissent être élevés dans un cadre culturel arménien, où l'on apprenait la langue et pratiquait la religion. Un lieu marginal donc, au cœur de la Turquie. D’une huitaine de pensionnaires àl'ouverture, le nombre, garçons et filles, atteint quatrevingt en 1961, raconte Garabet Orunöz, une soixantaine d'années, lui-même ancien de Kamp Armen. En 1962, la communauté s'installe à Tuzla, proche d'une cinquantaine de kilomètres d'Istanbul. Elle prospère sur un mode autogestionnaire, vergers et élevage - toute une époque -, jusqu'en janvier 1983 où elle est évacuée, suspectée d'être un lieu de «christianisation des musulmans »-alors qu'historiquement, c'est l'inverse qui s'est produit - et une enclave pour les terroristes de l'ASALA. Du fait d'un jeu de loi interdisant l'acquisition de propriété par les Arméniens, le bâtiment et le terrain, bien qu'acquis par un acte de vente, sont restitués à son précédent propriétaire. Güzelyan est arrêté, emprisonné huit mois. Après sa sortie, il choisit l'exil. Le dernier dimanche d'avril de chaque année, a lieu un pèlerinage pour entretenir cette histoire qui, depuis peu, risque d'être, elle aussi, définitivement effacée par les bulldozers afin d'implanter sur le terrain une zone résidentielle, avec la bénédiction des autorités locales et gouvernementales toujours prêtes à éradiquer les traces de l'arménité en Anatolie.

$$
\text { 棌淿 }
$$

La situation à Kamp Armen est régulièrement chroniquée dans Agos, cet hebdomadaire fondé en 1996 par Hrant Dink et un groupe d'amis. L'hebdomadaire a d'abord voulu rendre compte des problèmes que $\bullet \bullet$ 


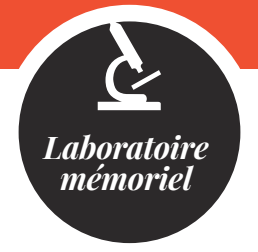

•• vivaient les Arméniens en Turquie et les faire connaître à la société. Il faut néanmoins rappeler que la liberté de parole est régie par une censure qui, même si son seuil de tolérance s'est récemment allégé, fait encourir un an de prison à ceux qui enfreignent le fameux article 301 du Code pénal en critiquant l'identité turque. Ce faisant, Agos a d'emblée été publié en turc et en arménien, événement sans précédent depuis la République (et depuis le génocide). Il devient l'organe d'une communauté qui restait silencieuse, contrainte et, à la fois, se contraignant à la discrétion de crainte d'entraîner des réactions de la part de l'État ou des milieux nationalistes. Mais Agos ne se dédie pas à n'être qu'un porte-parole communautaire, évitant ainsi le risque d'un repli sur soi. Le journal a maintenant 5000 lecteurs réguliers pour sa version papier, 10000 pour sa version électronique.

Sa ligne éditoriale s'est ouverte aux questions de démocratisation, de droits des minorités et d'une visibilité du passé dans lequel le génocide des Arméniens occupe une place centrale. "Agos ne se réduit pas à vouloir dénoncer la barbarie des crimes turcs », explique Yetvart Danzikyan, l'actuel rédacteur en chef du journal. Rien d'étonnant à ce que Nor Zartonk leur soit proche, de même que l'IHD, DurDe, Anadolu Kültur et tous ceux qui soutiennent un processus de

démocratisation cherchant, peu à peu, à infléchir l'irréductibilité des positions de l'État : la reconnaissance du génocide est en ce sens un levier qui dépasse les faits eux-mêmes et engage l'avenir de la Turquie par et dans le règlement des rapports avec sa propre histoire comme avec ses communautés. Aussi, s'agit-il, insiste toujours Danzikyan, de ne pas se refermer sur le passé mais, au contraire, de renforcer les liens avec la société civile, de susciter des prises de conscience et de rapprocher les différentes communautés. Cette ouverture n'a pas spontanément été bien acceptée par la communauté arménienne qui éprouvait aussi une sorte de peur de dévoiler ses « secrets », complète-t-il. Jusque dans quelle mesure les secrets - si douloureux soient-ils - ne peuvent-ils pas alimenter des identités de l'intérieur et y entretenir une sorte de culte du malheur? Paradoxalement, c'est en s'employant à rompre ce double mur, du déni du côté turc et du repli du côté des Arméniens de Turquie, que Hrant Dink a pris les risques qui ont entraîné son assassinat.

$$
\text { 氺皮 }
$$

La dernière rencontre dont je voudrais parler est en fait la première de ce court séjour. Je me retrouvais

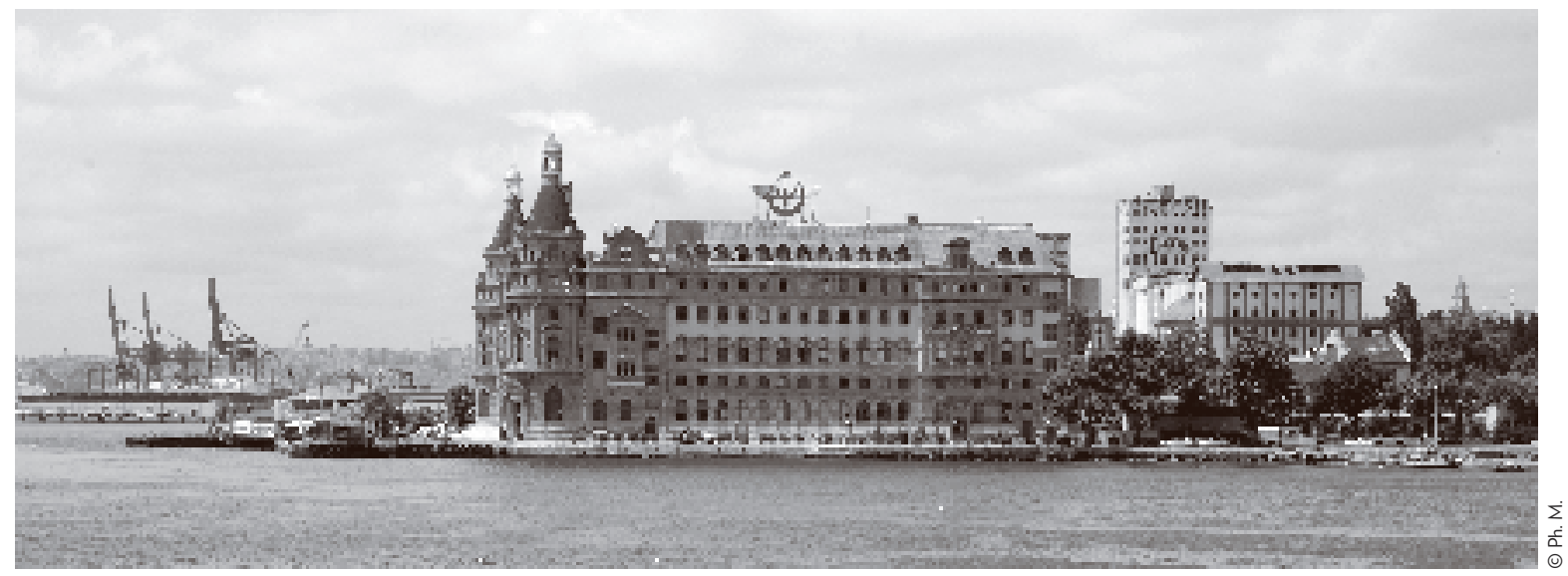

_ La gare de Haydarpaşa, aujourd'hui désaffectée, était la gare Centrale d'où partaient les trains vers l'Est du pays. C'est de là que les convois de déportation d'Arméniens d'Istanbul sont partis en 1915. (2015) 
au café Ara à proximité du Galatasaray Lisesi, certainement le plus célèbre lycée de Turquie. Burcin Gerçek est journaliste et, pratiquant parfaitement la langue française, elle est rédactrice dans la presse francophone et sur la plate-forme arméno-turque www.repairfuture.net. Burcin Gerçek nous fait découvrir une autre face de l'histoire du génocide qui s'impose aujourd'hui aux Turcs comme histoire à part entière de leur pays, à travers non seulement les révélations et les enquêtes de filiation ouvertes avec Le Livre de ma grand-mère de Fethiye Çetin (2004), mais aussi la reconnaissance que des Turcs, «intégralement turcs », ont sauvé des Arméniens durant la période du génocide. Certains ayant même été assassinés pour cette " trahison ». De qui s'agit-il? De cas isolés? - «ce sont des fonctionnaires, des chefs de tribu, des religieux et d'autres, tout à fait, ordinaires », précise-t-elle pour souligner que le mouvement n'était pas anodin, ni local. L'enquête que mène Burcin Gerçek est ardue. Les documents préservés ou accessibles, et dont une partie est écrite en caractères arabes, nécessitent impérativement d'être confrontés à la mémoire orale des descendants que Burcin Gerçek rassemble sur la base d'entretiens. En somme, ne sont-ils pas les équivalents de ceux que l'on nomme, en Europe, les Justes pour avoir sauvé des Juifs? «En fait, corrige-t-elle, il y a un problème terminologique. Il y a deux termes vicdanli ou hakkaniyetli qui se traduiraient par "consciencieux", celui qui a une conscience morale ». Précisément se concentre là un important enjeu de société que le pouvoir ne néglige pas.

De l'intérieur de la Turquie, explique Burcin Gerçek, la question ne peut seulement se poser dans les termes de la « reconnaissance du génocide des Arméniens ». En tant que telle, c'est d'une certaine manière le problème des politiques. Et si la société tout entière est concernée, la façon dont la reconnaissance l'interpelle ne peut se suffire. Le travail doit consister à mettre la population à portée d'un passé qui a été faussé de multiples façons depuis la fondation même de la République, et qui continue à l'être, insiste-t-elle. Les uns après les autres, les gouvernants s'obstinent à ne pas franchir le pas, à produire les discours et à soutenir les initiatives qui renforcent leur position. «Il en est ainsi de l'Adil Hafiza, dite "mémoire juste" qui, portée par l'argument du "nous avons tous souffert", tâche précisément de récupérer ceux que l'on nommerait en Europe des "Justes" ». Le gouvernement construit lui aussi sa mémoire - une contre-mémoire qui exalte le nationalisme en utilisant notamment les commémorations du centenaire de la Première Guerre mondiale, par exemple à Gallipoli où les armées australienne et néo-zélandaise ont essuyé une terrible défaite en 1915, l'année même où les Arméniens étaient déportés et massacrés.

Les sauveteurs d'Arméniens racontent d'une autre façon l'histoire du génocide. «Car si des Turcs ont pris des risques pour sauver des Arméniens, pourquoi aujourd'hui ne pas reconnaitre ces faits? », avance Burcin Gerçek. «La question est d'ouvrir aux Turcs une autre perspective pour qu'ils se mettent différemment en rapport avec cette histoire sans passer par une vision officielle qui soude l'unité nationale, malgré le contrekémalisme des récents gouvernements. Il s'agirait de sortir de cette interprétation monolithique et, par là même, fragile devant toute altérité dont elle se sent menacée, a fortiori si elle la conçoit comme altérité de l'intérieur. » I

Philippe Mesnard

Toute notre gratitude à Sila Cehreli pour nous avoir guidés à travers Istanbul comme à travers notre enquête.

\section{BIBLIOGRAPHIE}

- Akçam, Taner, 2012, Un acte honteux. Le génocide arménien et la question de la responsabilité turque [2008], Paris, [Denöel] Gallimard, « essai folio ".

- Özdamar, Emine Sevgi, 1998, Mutterzunge, Cologne, Kiepenheuer \& Witsch.

- Marchand, Laure \& Perrier, Guillaume, 2013, La Turquie et le fantôme arménien, préface de Taner Akçam, Arles, Actes Sud.

- Marian Michel, 2015, Le Génocide arménien. De la mémoire outragée à la mémoire partagée, Paris, Albin Michel, «Bibliothèque Idées ».

- Üngör, Uğur Ümit, avril 2015, « Mémoire, génocide et identité ». Témoigner entre histoire et mémoire, Paris, Kimé, $n^{\circ}$ 121, p. 57-66.

- Renan, Ernest, 1997, Qu'est-ce qu'une nation?, Paris, Mille et une nuits. 